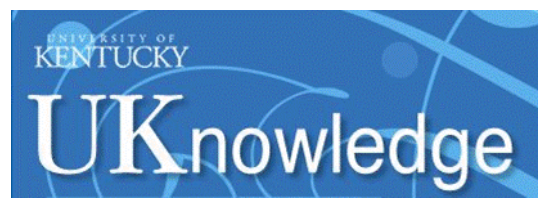

University of Kentucky

UKnowledge

6-2013

\title{
A New Approach To Light-Weight Ablators Analysis: From Micro- Tomography Measurements to Statistical Analysis and Modeling
}

\author{
Nagi N. Mansour \\ NASA Ames Research Center \\ Francesco Panerai \\ University of Kentucky, francesco.panerai@uky.edu \\ Alexandre Martin \\ University of Kentucky, alexandre.martin@uky.edu \\ Dilworth Y. Parkinson \\ Lawrence Berkeley National Laboratory \\ Alastair A. MacDowell \\ Lawrence Berkeley National Laboratory
}

See next page for additional authors

Follow this and additional works at: https://uknowledge.uky.edu/me_facpub

Part of the Aerospace Engineering Commons

Right click to open a feedback form in a new tab to let us know how this document benefits you.

\section{Repository Citation}

Mansour, Nagi N.; Panerai, Francesco; Martin, Alexandre; Parkinson, Dilworth Y.; MacDowell, Alastair A.; Haboub, Abdelmoula; Sandstrom, Timothy A.; Fast, Tony; Vignoles, Gerard L.; and Lachaud, Jean, "A New Approach To Light-Weight Ablators Analysis: From Micro-Tomography Measurements to Statistical Analysis and Modeling" (2013). Mechanical Engineering Faculty Publications. 6.

https://uknowledge.uky.edu/me_facpub/6

This Conference Proceeding is brought to you for free and open access by the Mechanical Engineering at UKnowledge. It has been accepted for inclusion in Mechanical Engineering Faculty Publications by an authorized administrator of UKnowledge. For more information, please contact UKnowledge@lsv.uky.edu. 


\section{A New Approach To Light-Weight Ablators Analysis: From Micro-Tomography Measurements to Statistical Analysis and Modeling}

Digital Object Identifier (DOI)

http://dx.doi.org/10.2514/6.2013-2768

Notes/Citation Information

Published in the Proceedings of the 44th AIAA Thermophysics Conference, Paper 2013-2768, p. 1-11.

This material is declared a work of the U.S. Government and is not subject to copyright protection in the United States.

\section{Authors}

Nagi N. Mansour, Francesco Panerai, Alexandre Martin, Dilworth Y. Parkinson, Alastair A. MacDowell, Abdelmoula Haboub, Timothy A. Sandstrom, Tony Fast, Gerard L. Vignoles, and Jean Lachaud 


\title{
A New Approach To Light-Weight Ablators Analysis: From Micro-Tomography Measurements to Statistical Analysis and Modeling
}

\author{
Nagi N. Mansour* \\ NASA Ames Research Center, Moffett Field, CA, 94035 \\ Francesco Panerai ${ }^{\dagger}$ and Alexandre Martin ${ }^{\ddagger}$ \\ University of Kentucky, Lexington, KY, 40506-0503
}

Dilworth Y. Parkinson ${ }^{\S}$, Alastair A. MacDowell ${ }^{\S}$ and Abdelmoula Haboub

Lawrence Berkeley National Laboratory, Berkeley, CA, 94720

Timothy A. Sandstrom"

NASA Ames Research Center, Moffett Field, CA, 94035

Tony Fast**

Gerard L. Vignoles ${ }^{\dagger \dagger}$

Georgia Institute of Technology, Atlanta, GA, 30332

Université Bordeaux, 33600 Pessac, France

\author{
and Jean Lachaud $\mathrm{d}^{\ddagger}$ \\ UC Santa Cruz, Moffett Field, CA, 94035
}

\begin{abstract}
The morphology characteristics and ablation behavior of a highly porous carbon fiber preform are studied using a combined experimental/numerical approach. Morphological characterization of the three-dimensional structure of the material is performed by hard X-rays synchrotron micro-tomography at the Advanced Light Source of Lawrence Berkeley National Laboratory. The resulting micro-tomography voxels are used to compute geometrical properties of the carbon preform, like porosity, specific surface area and tortuosity, that are otherwise indirectly measured through experimental techniques. The reconstructed volumes are used to build a computational grid for numerical simulations of the fibers' ablation. By modeling the diffusion of oxygen through the porous medium using Lagrangian methods, and the oxidation at the carbon fibers' surface using a reactivity model, the ablation of the carbon fibers are simulated for a range of Thiele numbers. It is shown that in the diffusion limited regime (large Thiele number), the ablation of the fibers occurs at the surface of the material. In the reaction limited regime (low Thiele number), the oxygen penetrates into the fibers, resulting in volumetric ablation and high material spallation.
\end{abstract}

${ }^{*}$ Chief Division Scientist, NASA Advanced Supercomputing Division, Mail Stop 258-5, AIAA Associate Fellow.

$\dagger$ Postdoctoral Scholar, Department of Mechanical Engineering, 261 Ralph G. Anderson Building. Visiting Scientist, Thermal Protection Materials Branch, Mail Stop 234-1, NASA Ames Research Center, Moffett Field, CA, 94035, AIAA Senior Member.

${ }^{\ddagger}$ Assistant Professor, Department of Mechanical Engineering, Associate Faculty at the Center for Computational Science, 261 Ralph G. Anderson Building, AIAA Senior Member.

$\S$ Beamline Scientist, Advanced Light Source, 1 Cyclotron Road, Mail Stop 2-400.

TPostdoctoal Fellow, Advanced Light Source, 1 Cyclotron Road, Mail Stop 2-400.

"Senior Research Scientist, NASA Advanced Supercomputing Division, Mail Stop 258-5.

** Research Scientist, Mosaic of Microstructures MURI.

$\dagger^{\dagger}$ Professor, Lab. des Composites ThermoStructuraux (LCTS), 3, Allée La Boëtie.

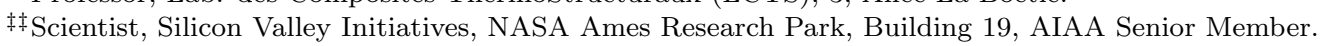




\section{Introduction}

Lightweight carbon/phenolic ablators are a new class of low density composites that are used as thermal protection materials for high speed planetary entry. This class of materials is usually made by impregnating a carbon preform substrate with phenolic. The Phenolic Impregnated Carbon Ablator (PICA) invented by NASA uses Fibeform ${ }^{\circledR}$ as a substrate. Fibeform ${ }^{\circledR}$ is an industrial carbon preform developed by Fiber Material Inc. (Biddeford, ME, USA) for insulation of industrial furnaces. PICA, manufactured by FMI for NASA, has demonstrated outstanding performance during Earth atmosphere entry of the Stardust sample return capsule, and Mars atmosphere entry of the Mars Science Laboratory capsule that successfully landed the Curiosity rover on the surface of Mars. PICA-X, a variant of PICA developed by SpaceX (Space Exploration Technologies Corp., Hawthorne, CA, USA), has been successfully used on the Dragon capsule, the first commercial vehicle in history to service the International Space Station.

A critical requirement in sizing the material on a thermal protection systems (TPS) is the ability of the designer to estimate the material response to high-enthalpy environments. A reliable model, needed to predict bondline temperatures and surface recession under high-enthalpy entry conditions, is needed to optimize the heat-shield thickness and provide margins. Material response models, currently used in TPS design, treat the ablation of PICA as a surface phenomenon, and assume that under high enthalpy environments, material mass is lost at the surface. The pyrolysis of the bulk phenolic matrix is modeled, but the in-depth density of the resulting char is assumed constant. To reduce design margins and improve the mass budget requirement for the TPS, phenomenological models accounting for the micro-structure of the material are under development. ${ }^{1-4}$ Understanding and modeling the response of carbon preforms, the basic component of many carbon/phenolic ablators, is a first step towards validation of this new generation of material response mod-

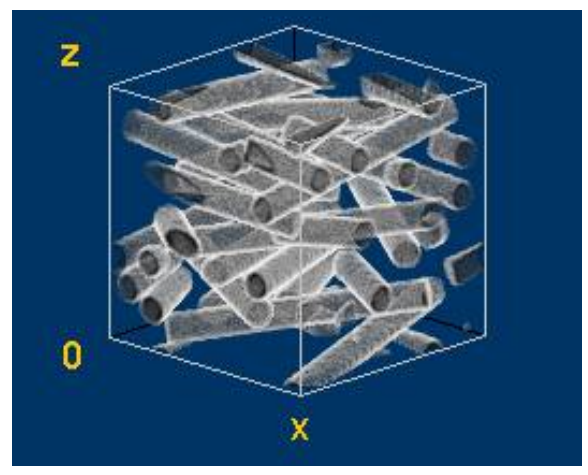

Figure 1. Artificial model of carbon preform built by Monte Carlo algorithm, from Ref. 1 els.

In a previous work, microscopic simulations on carbon preforms were performed by digitally building virtual materials (see Fig. 1). ${ }^{1,3}$ This digital fibrous material, represented on a Cartesian grid as a cube of voxels, was built using a Monte Carlo algorithm to fill the volume in which non-overlapping fibers, with random positions and orientations, were positioned until a desired porosity target level was reached. To obtain a realistic reproduction of the material's architecture, fibers were constructed to have their direction azimuthally biased to be at small angles $\left(<|15|^{\circ}\right)$ with respect to the top surface boundary. This constraint was used to match the physical specifications of FiberForm ${ }^{\circledR}$.
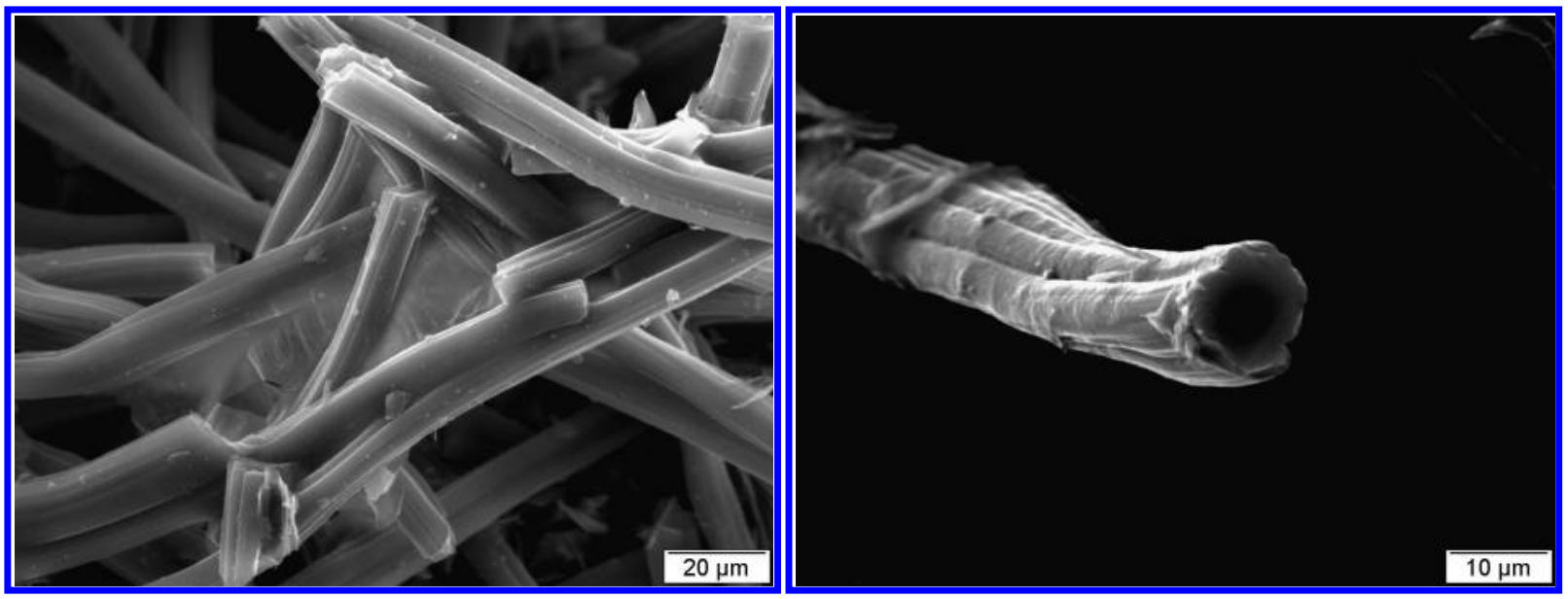

Figure 2. Scanning electron micrographs of virgin carbon preform at different magnification levels.

Comparing Fig. 1 to the high resolution Scanning Electron Microscope (SEM) images of FiberForm ${ }^{\circledR}$ in 
Fig. 2, clearly shows that the digitized material oversimplifies the actual structure of the fibers. One can notice that the architecture and morphology of the digital material lack many features. The manufacturing process of carbon preforms consists of bonding fibers together by means of a phenolic resin former. The fiberformer combination is fully carbonized at high temperature, yielding a porous rigid composite. Micrographs show that carbon bonds are left at the fibers' intersections at several locations throughout the sample. These clumps of carbon are not modeled in the digital material. Moreover, high magnification scans show that what is usually modeled as a fiber is actually a strand of multiple fibers joined together. The geometry of the actual fibers is far from a perfect cylinder as it is used in building the digital material.

The possibility to obtain tomographs of the actual carbon preform material represents a major advancement in our ability to analyze physical characteristics and ablation behavior of this material. In section II, X-ray micro-tomography ${ }^{5}$ is used to image the fiber-scale three-dimensional morphology of FiberForm ${ }^{\circledR}$. We demonstrate in section III that these X-ray tomographs can be used to characterize relevant physical properties of the material such as volume fraction, specific surface area and tortuosity. Finally, in section IV, we show that these micro-tomography voxels can be directly used as the discretized geometry of the actual material in AMA, ${ }^{6}$ a code that models diffusion/reaction, to simulate the microscopic-scale oxidation and ablation of carbon preform. We conclude the paper in section $\mathrm{V}$, with future plans for the use of the X-ray tomography technology to enable advancements in modeling material response to high-enthalpy environments.

\section{X-ray Micro-tomography Measurements}

Hard X-ray micro-tomography measurements were performed at Beamline 8.3.2 at the Advanced Light Source (ALS) of Lawrence Berkeley National Laboratory. ${ }^{5}$ X-ray tomography (or computed tomography, $\mathrm{CT}$ ) exploits the capability of different materials or phases to absorb X-ray radiation, due to their different density and chemical composition. The synchrotron source provides a high density X-ray flux, allowing the resolution of micron and sub-micron features with low noise and high quality.

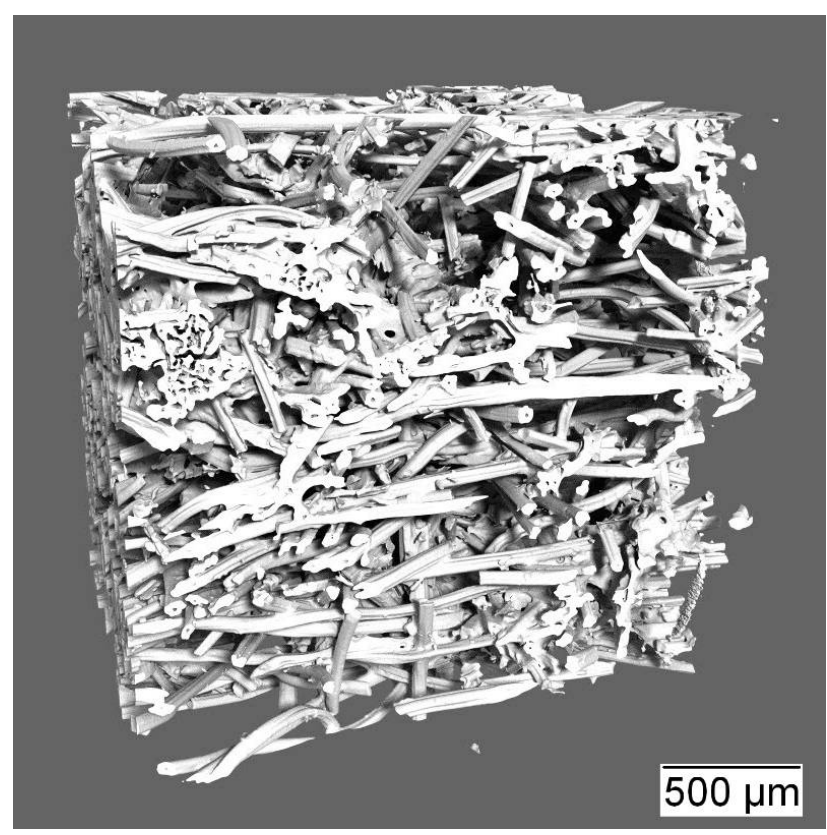

Figure 3. Visualization of carbon preform extracted from a tomography scan. interface developed in-house at the ALS, that links to the Octopus software (inCT, Aalst, Belgium) ${ }^{11}$ for tomographic reconstruction. The rendering was performed using a Monte Carlo ray tracing technique based on the path tracing algorithm ${ }^{12}$ implemented in the Embree kernel (Intel Corp., Santa Clara, CA, USA). 


\section{Statistical Analysis of the morphology of carbon fiber preform}

Having a digitized 3D image of the material wherein each voxel has a grayscale value between 0 (black) and 255 (white), statistical analysis on the morphology of the material can be performed. Note that in the raw data the void is represented as black and the carbon is represented as white. Figure 4 shows the normalized distribution (histogram) of the raw pixel values. The sharp leveling of the distribution curve, labeled "Cutoff Region" on the figure, is the region where voxel values transition from void to fiber. The interface between void pixels and fiber pixels is around a gray-scale value of 80 .

By binning the material as void for pixels below the cutoff and fiber above the cutoff, the volume fraction of the fibers can be computed as a function of the pixel value cutoff (see insert Fig. 4). The computed value of the volume fraction of the fibers is $20 \%$ which is very close to effective density measurements and the manufacturer specification of FiberForm ${ }^{\circledR}(12.8 \% \pm 7)$.

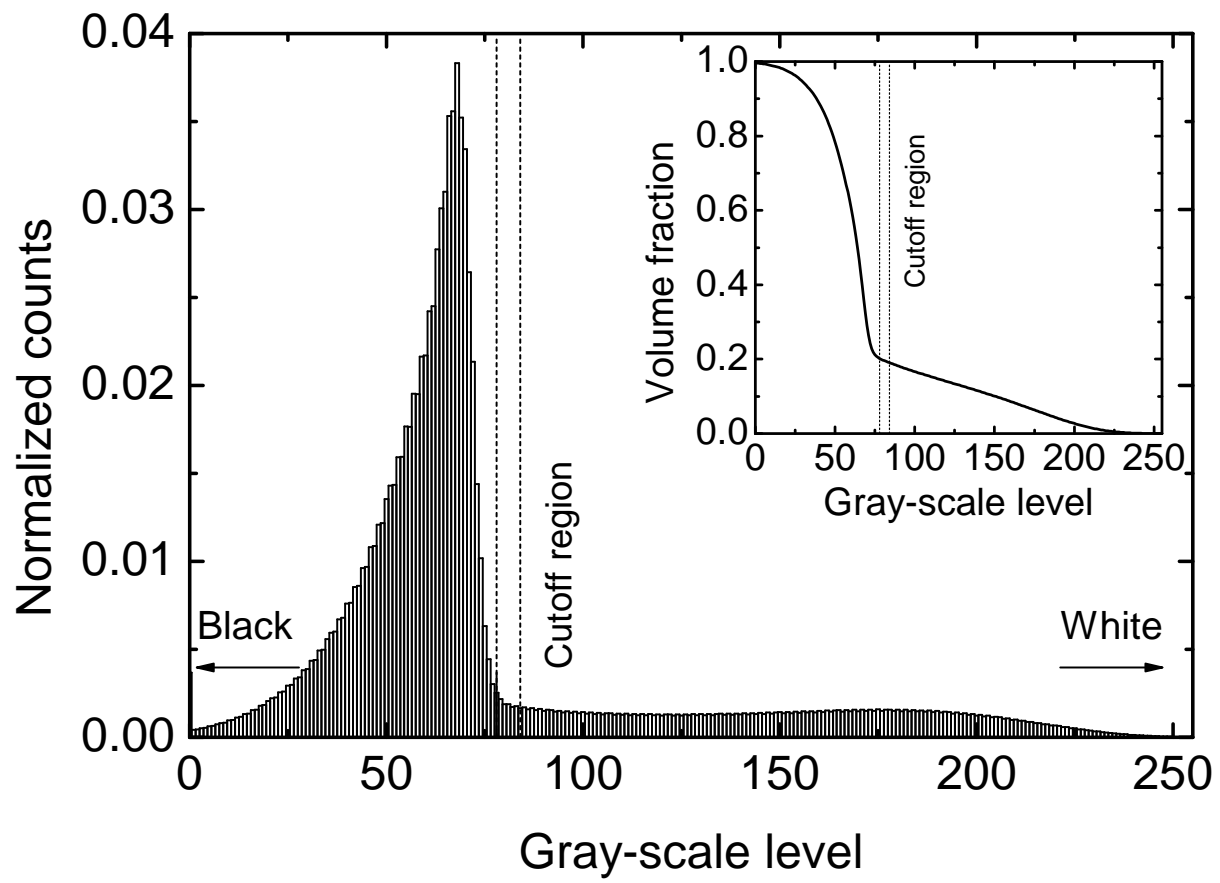

Figure 4. Normalized Count (histogram), , $N C$, of the pixel values in the raw sample 3D image as a function of the gray scale level, GSL. The insert is the volume fraction computed as $1-\sum_{0}^{G S L} N C$

Two quantities of importance in modeling porous materials are the specific surface area (SSA) of the interface and the tortuosity of the material. Figure 5 shows the SSA as a function of the pixel cutoff. We find that the curve levels off quickly around a pixel value of 80 , again indicating that the interface for thresholding between void and fibers can be defined with confidence.

The tortuosity is defined to be the distance of the shortest path that a particle would travel through the void from one surface of a volume to its opposite surface, normalized by the distance between the two surfaces. Figure 6 shows the distribution of the shortest path to length for a coarse version of our sample computed for each surface pixel using graph based methods. ${ }^{13}$ We find the peak value of the geometrical tortuosity to be around around 1.5 in the z-direction and 1.3 in the $\mathrm{x}$ - and y-directions (i.e in plane normal to the z-direction). This is due to the fact that the directions of the fibers in FiberForm ${ }^{\circledR}$ are not isotropic, but they are randomly oriented within $\pm 15^{\circ}$ from a bias plane. Given a position on a plane, there are several pathways to reach the opposite plane, we have also plotted in Fig. 6 the average path between two planes. We find that in this measure the material looks more isotropic, but the shortest path is the most relevant quantity for computing the conduction properties. 


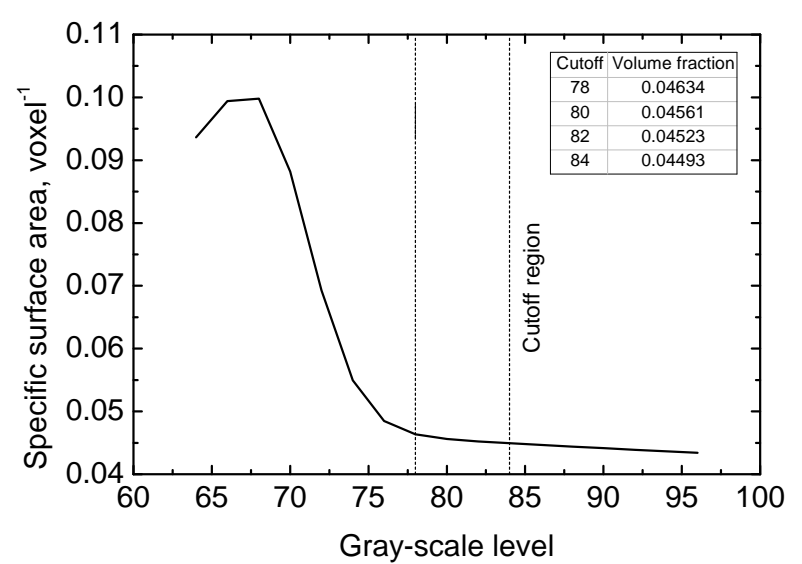

Figure 5. Specific surface area of carbon fibers as a function of the choice of pixel value cut-off for binning.

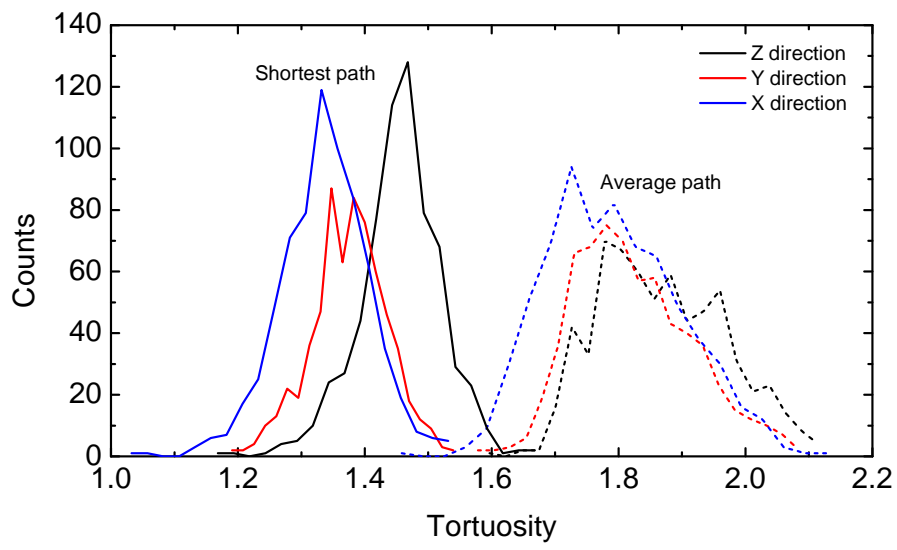

Figure 6. Distribution of the shortest and average path from one side of the volume to its opposite side, computed using a cutoff pixel value of 80 .

\section{Microscopic Ablation Model}

Using the 3D tomographs, the oxidation of the carbon fibers is simulated using the AMA algorithm, described in Ref. 6 . The method is based on a Monte-Carlo random-walk principle to simulate mass transfer from continuum to Knudsen regimes and provides a three-dimensional time-dependent solution of the reaction/diffusion problem coupled to surface recession, based on a pseudo-VOF algorithm with a Simplified Marching Cubes $^{14}$ interface discretization.

\section{A. Summary of the AMA algorithm}

The first step in the algorithm consists of generating the computational domain by binning the cubic voxels. In the present study the grid is generated by setting the voxel value of 80 to be the threshold for the voxelbased tomography images. A $100 \times 100 \times 500$ brick is extracted from the $1600^{3}$ cubic dataset presented in Fig. 3, corresponding approximately to a $0.13 \times 0.13 \times 0.65 \mathrm{~mm}$ volume.

The ablation processes, defined as non-mechanical removal of matter by physico-chemical processes, is modeled by a moving fluid/solid interface, the position of which is defined by $S(x, y, z, t)$. The normal velocity $\vec{v}_{r}$ of the receding front is set to be proportional to the reactivity of the fibers. A Simplified Marching Cubes ${ }^{14}$ algorithm is used to mesh the interface. The interface movement obeys the following Hamilton-Jacobi differential equation:

$$
\frac{\partial S}{\partial t}+\vec{v}_{r} \nabla \partial S=0
$$

Equation 1 describes the recession of the surface in an Eulerian frame. The recession velocity can be written as

$$
\vec{v}_{r}=\Omega \cdot J_{i} \vec{n}=\Omega C_{i} k_{f} \vec{n}
$$

where $\vec{n}$ is the normal unit vector to the surface, $\Omega$ is the solid molar volume and $J_{i}$ is the molar flux of species $i$, that, for a first order heterogeneous reaction, can be expressed as the product of the species $i$ concentration $C_{i}(x, y, z, t)$ and the fiber reactivity $k_{f}$. Ablation due to oxidation by molecular oxygen $\left(i=\mathrm{O}_{2}\right)$ is considered in this work.

The mass transfer by diffusion is simulated by a random walk using Maxwell-Boltzmann distribution for the free path in the Knudsen and transition regimes, and a Brownian motion simulation technique in the continuum and slip regimes. ${ }^{3}$ The conservation of oxygen concentration in the fluid phase can be written as:

$$
\frac{\partial C_{\mathrm{O}_{2}}}{\partial t}+\nabla \cdot\left(D \nabla C_{\mathrm{O}_{2}}\right)=0
$$

where $\mathrm{D}$ is the diffusion coefficient (binary diffusion coefficient of oxygen in air). 

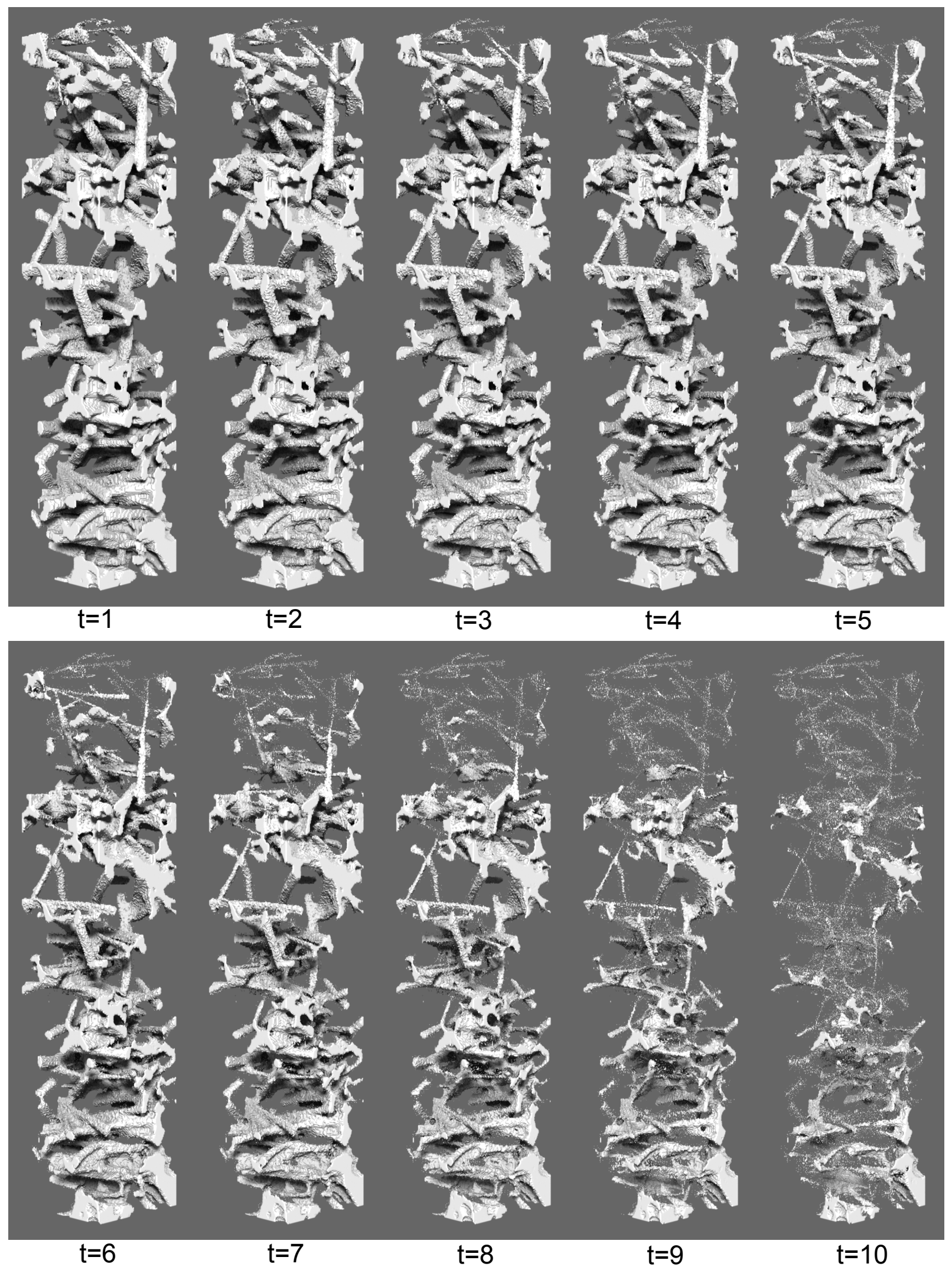

Figure 7. AMA simulations of the progressive oxidation of the carbon fibers at low Thiele number $(\Phi<0.05)$. The computational time $t$ is in arbitrary units (a.u.). 

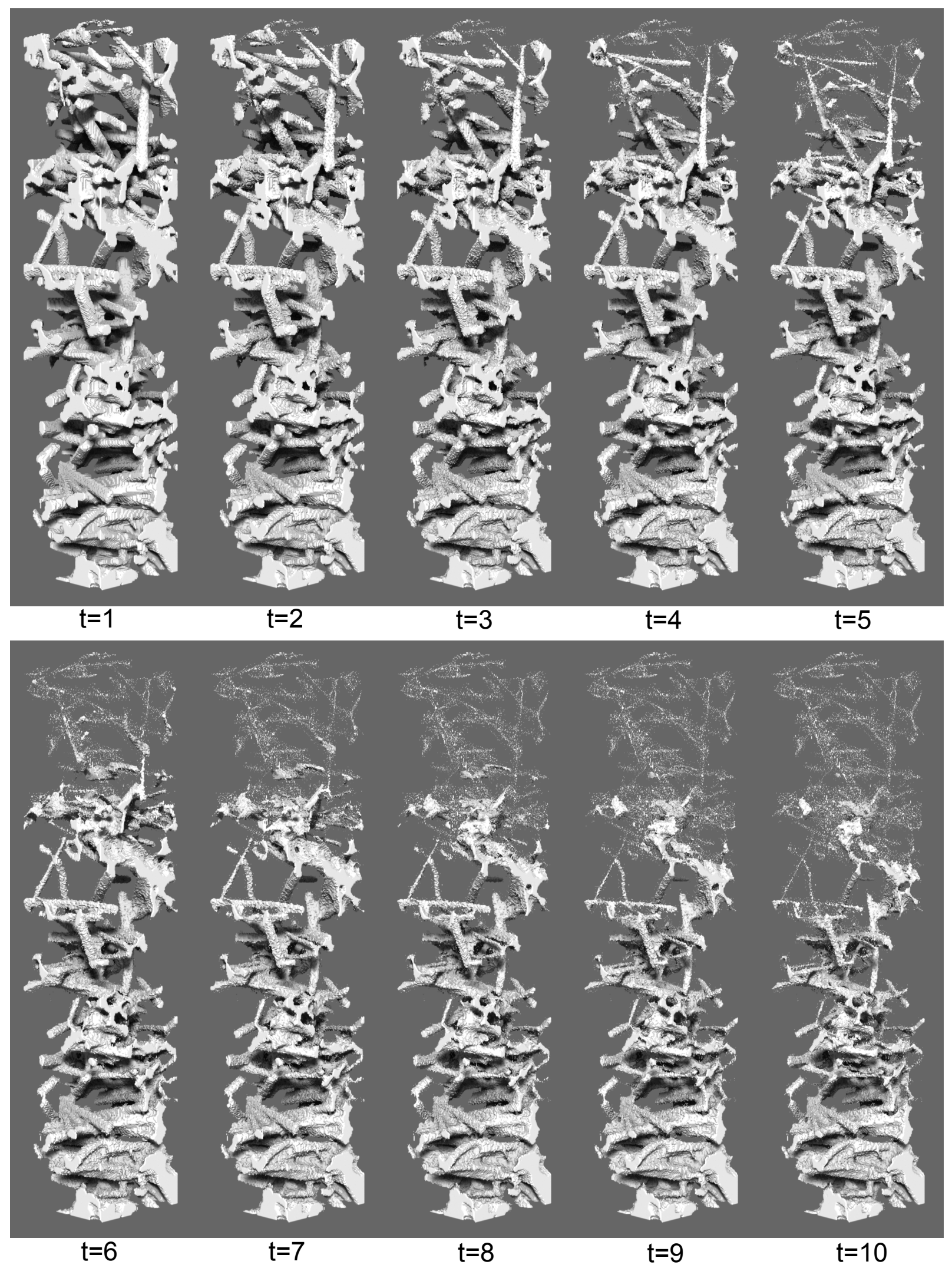

Figure 8. AMA simulations of the progressive oxidation of the carbon fibers at intermediate Thiele number $(\Phi \sim 1)$. The computational time $t$ is in arbitrary units (a.u.). 


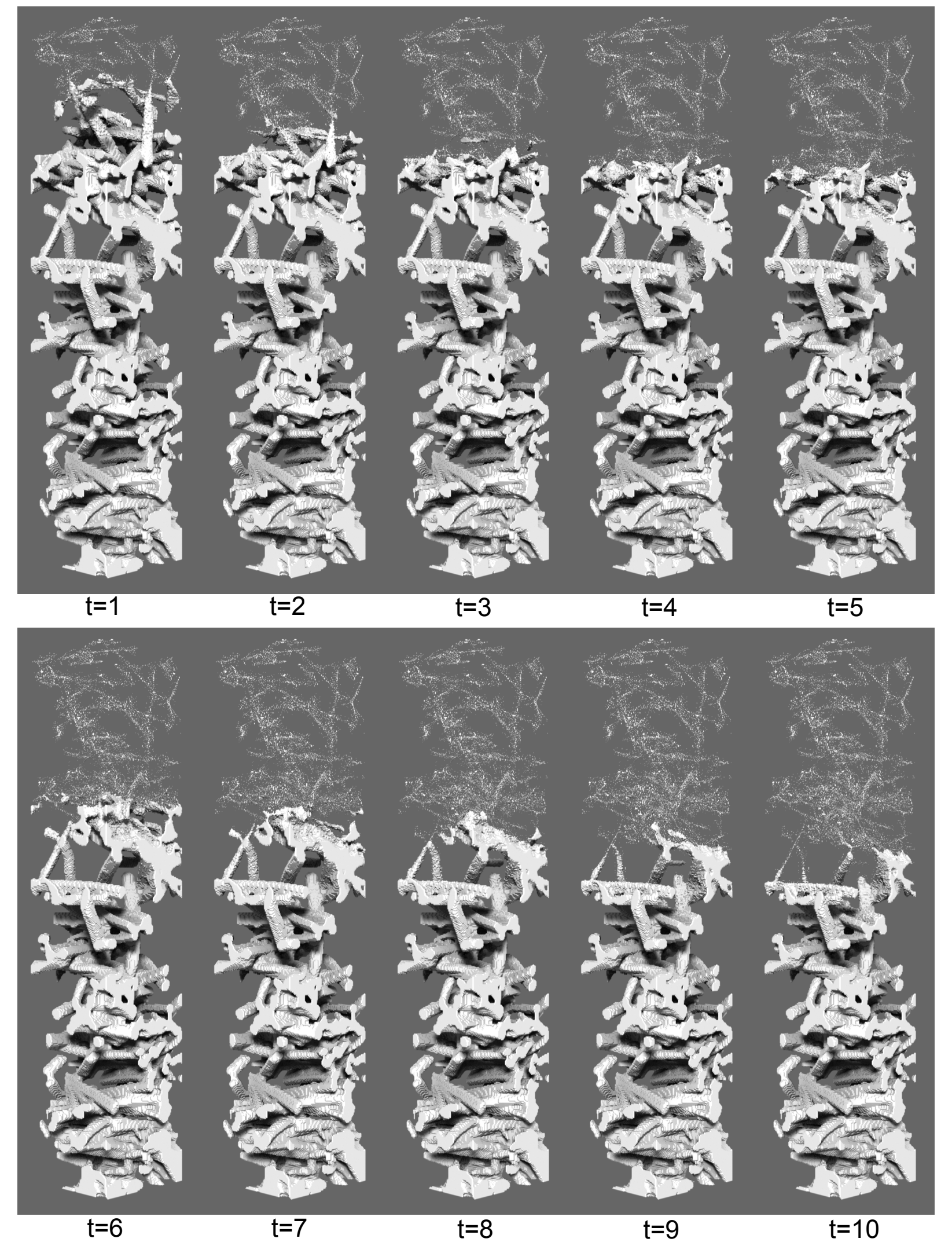

Figure 9. AMA simulations of the progressive oxidation of the carbon fibers at high Thiele number $(\Phi>50)$. The computational time $t$ is in arbitrary units (a.u.). 


\section{B. Simulation Results}

Three simulations of the oxidation behavior of the reconstructed carbon preform were performed. Figures 7 , 8 and 9 show the simulated oxidation progression, at low $(\Phi<0.05)$, intermediate $(\Phi \sim 1)$ and high $(\Phi>50)$ Thiele number values respectively. The Thiele number is the dimensionless parameter that describes the competition between reactant diffusion and surface reactivity in a porous material. It can be written as:

$$
\Phi=\frac{L}{\left(\mathcal{D}_{\text {eff }} / s_{f} k_{f}\right)^{1 / 2}}
$$

where $\mathrm{L}$ is a characteristic length scale of the system, $\mathcal{D}_{\text {eff }}$ is the effective diffusion coefficient, $s_{f}$ is the specific surface area of the porous medium and $k_{f}$ is the fiber reactivity. In the case of ablation by oxygen transport, high Thiele numbers characterize a diffusion-limited situation, where ablation occurs mostly at the interface between the fibers and the incoming flow. Ablation in this case is a surface phenomenon. Conversely, at low Thiele numbers, the oxidation is reaction-limited, oxygen can penetrate in depth and an ablation zone large compare to the fiber diameter can be identified. Ablation in this case is a volumetric process. At intermediate Thiele numbers, the length scales of diffusion and reaction are similar and the depth of the reaction zone is of the order of the fiber diameter.

Numerically, the present simulations are similar to those previously performed on the digital carbon-fiber material shown in Fig. $1 .^{3}$ These simulations replicate flow-tube reactor experimental conditions aimed at measuring the reactivity of carbon fibers. ${ }^{4}$ A fixed oxygen concentration is imposed at the top boundary, and a no-flux boundary condition is imposed at the bottom of the cell. In flow-tube reactor experiments a plug (cylinder) sample of carbon preform is heated to a certain temperature (typically between 700 and $1300 \mathrm{~K}$ ), while it is submitted on one face to a flow of air (containing oxygen reactants), and the other faces are protected from the fresh airflow. ${ }^{4}$ Reference 4 documents results obtained, with such a configuration, at $898 \mathrm{~K}$ and $12.8 \mathrm{kPa}$. Reactor experiments performed on hollow cylindrical models ${ }^{15}$ show that varying pressure conditions from $\sim 1$ to $\sim 100 \mathrm{kPa}$, enables varying the Knudsen number, within the porous medium, from the transitional, to the slip and then to the continuum regime. A modeling effort to simulate those experiments is also proposed in a companion paper at this conference. ${ }^{16}$

Figure 8 , at a Thiele number of $\sim 1$, presents the intermediate case where the oxidation/diffusion competition is fully active. Oxygen diffuses in the preform but, at the same time, it is quickly consumed by the oxidation reaction of the carbon fibers. Oxygen penetration is low and, as a result, after ten computational time units $(t=10)$ the depths of the oxidation zone is about $400 \mu \mathrm{m}$. The dusts observed in the images are numerical artifacts of the Simplified Marching Cubes method, by slightly adjusting the contour levels we could have eliminated them from the figures. Addressing the accuracy of the numerical method is not within the scope of this paper and will be discussed elsewhere. We have kept the dust in the images to help the reader visualize where the material existed.

The two extreme cases reproduce respectively a reaction-limited volumetric oxidation (Fig. 7, the low $\Phi$ case) and a diffusion-limited surface oxidation (Fig. 9, the high $\Phi$ case). Consequently, at $t=10$ computational time units, for $\Phi<0.05$ the ablated region covers nearly the full length of the computational domain $(\sim 650 \mu \mathrm{m})$, while for $\Phi>50$, the oxidation depth is only $\sim 300 \mu \mathrm{m}$ (see Fig. 10).

\section{Conclusion}

We have demonstrated the unique capability that tomography will enable in this branch of material science. In our particular application of interest where the material is highly porous, having a digitized representation of the material enables direct measurements of material properties such as porosity, specific surface area and tortuosity. Future work will show that the anisotropy of the heat conductivity of FiberForm ${ }^{\circledR}$ can be estimated from the voxels. In addition, estimates of Darcy's permeability coefficient can also be obtained.

We have also demonstrated that having access to the micro-structure of the material enables realistic simulations of material response to oxidation. Not discussed in this paper but will be a subject of a future paper, the AMA methodology enables estimates of material spallation, a mechanical path for TPS material loss. Modeling material spallation has been a challenge that has eluded proper modeling in the past. 


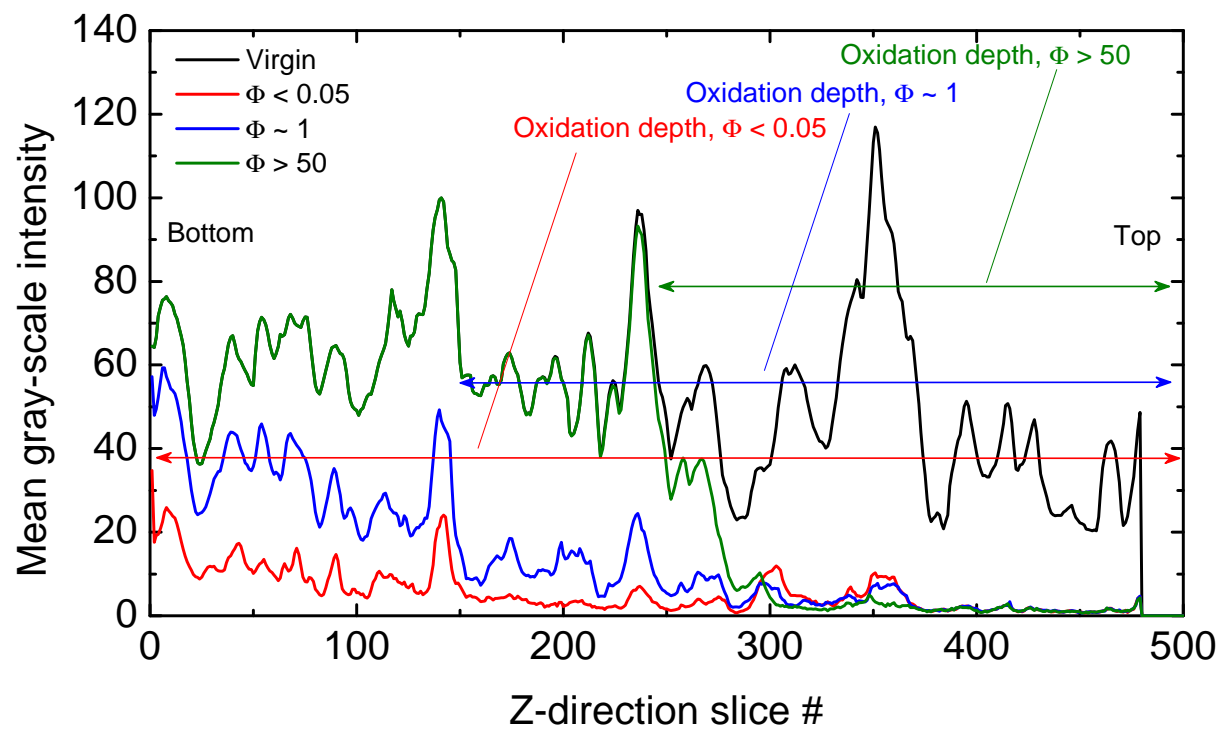

Figure 10. Oxidation zone depth at $t=10$ for different Thiele regimes, as measured by horizontally averaging the gray scale. A slice \# is equivalent to $1.3 \mu \mathrm{m}$.

\section{Acknowledgement}

This effort was partially supported by the Fundamental Aeronautics hypersonics EDL project. We gratefully acknowledged the support and encouragements of Drs. M.J. Wright and A.M. Calomino during the course of this research. Financial support to F.P. was provided by NASA SBIR Phase-2 Award NNX10CC53P, and NASA Kentucky EPSCoR Award NNX10AV39A. The Advanced Light Source is supported by the Director, Office of Science, Office of Basic Energy Sciences, of the U.S. Department of Energy under Contract No. DE-AC02-05CH11231.

\section{References}

${ }^{1}$ Lachaud, J., Cozmuta, I., and Mansour, N., "Multiscale Approach to Ablation Modeling of Phenolic Impregnated Carbon Ablators," Journal of Spacecraft and Rockets, Vol. 47, No. 6, 2010, pp. 910-921.

${ }^{2}$ Mansour, N., Lachaud, J., Magin, T., de Mûelenaere, J., and Chen, Y.-K., "High-Fidelity Charring Ablator Thermal Response Model," 42 $2^{\text {nd }}$ AIAA Thermophysics Conference, AIAA 2011-3124, Honolulu, HI, USA, 2011.

${ }^{3}$ Mansour, N., I., and Lachaud, J., "Microscopic Scale Simulation of the Ablation of Fibrous Materials," $48^{\text {th }}$ AIAA Aerospace Sciences Meeting Including the New Horizons Forum and Aerospace Exposition, AIAA 2010-984, Orlando, FL, USA, 2010

${ }^{4}$ Lachaud, J., Mansour, N., Ceballos, A., Pejaković, D., Zhang, L., and Marschall, J., "Validation of a Volume-Averaged Fiber-Scale Model for the Oxidation of a Carbon-Fiber Preform," 42 ${ }^{\text {nd }}$ AIAA Thermophysics Conference, AIAA 2011-3640, Honolulu, HI, USA, 2011.

${ }^{5}$ MacDowell, A. A., Parkinson, D. Y., Haboub, A., Schaible, E., Nasiatka, J. R., Yee, C. A., Jameson, J. R., Ajo-Franklin, J. B., Brodersen, C. R., and McElrone, A. J., "X-ray micro-tomography at the Advanced Light Source," Proc. SPIE 8506, Developments in X-Ray Tomography VIII, 2012, pp. 850618-14.

${ }^{6}$ Lachaud, J. and Vignoles, G., "A Brownian Motion Technique to Simulate Gasification and its Application to C/C Composite Ablation," Computational Materials Science, Vol. 44, No. 6, 2009, pp. 1034-1041.

${ }^{7}$ Kak, A. and Slaney, M., Principles of Computerized Tomographic Imaging, IEEE Press, 1988.

${ }^{8}$ Cloetens, P., Barrett, R., Baruchel, J., Guigay, J., and Schlenker, M., "Phase objects in synchrotron radiation hard-X-ray imaging," J. Phys. D, Vol. 29, 1996, pp. 133-146.

${ }^{9}$ Paganin, D., Mayo, S., Gureyev, T., Miller, P., and Wilkins, S., "Simultaneous Phase and Amplitude Extraction from a Single Defocused Image of a Homogeneous Object," Journal of Microscopy, Vol. 206, No. 1, 2002, pp. 33-40.

${ }^{10}$ Schindelin, J., Arganda-Carreras, I., Frise, E., Kaynig, V., Longair, M., Pietzsch, T., Preibisch, S., Rueden, C., Saalfeld, S., Schmid, B., Tinevez, J.-Y., White, D. J., Hartenstein, V., Eliceiri, K., Tomancak, P., and Cardona, A., "Fiji: an Open-Source Platform for Biological-Image Analysis," Nature Methods, Vol. 9, No. 7, 2012, pp. 676-682.

${ }^{11}$ Dierick, M., Masschaele, B., and Van Hoorebeke, L., "Octopus, a fast and user-friendly tomographic reconstruction package developed in LabView," Measurement Science and Technology, Vol. 15, No. 7, 2004, pp. 1366.

${ }^{12}$ Kajiya, J. T., "The rendering equation," SIGGRAPH Comput. Graph., Vol. 20, No. 4, Aug. 1986, pp. $143-150$. 
${ }^{13}$ Çeçen, A., Wargo, E. A., Hanna, A. C., Turner, D. M., Kalidindi, S. R., and Kumbur, E. C., "3-D Microstructure Analysis of Fuel Cell Materials: Spatial Distributions of Tortuosity, Void Size and Diffusivity," .

${ }^{14}$ Donias, M., Vignoles, G. L., Mulat, C., Germain, C., and Delesse, J.-F., "Simplified Marching Cubes : an efficient discretization scheme for simulations of deposition/ablation in complex media," Computational Materials Science, Vol. 50, 2011, pp. 893-902.

${ }^{15}$ Panerai, F., Martin, A., Mansour, N., Sepka, S., and Lachaud, J., "Flow-tube Oxidation Experiments on the Carbon Preform of PICA," $44^{\text {th }}$ AIAA Thermophysics Conference, San Diego, CA, USA, 2013.

${ }^{16}$ Martin, A., "Volume Averaged Modeling of the Oxidation of Porous Carbon Fiber Material." $44^{\text {th }}$ AIAA Thermophysics Conference, San Diego, CA, USA, 2013. 